OPEN ACCESS

University of Dundee

\title{
Asymptotic solutions for laminar flow based on blood circulation through a uniformlyporous channel with retractable walls and an applied transverse magnetic field
}

Li, Lin; Lin, Ping; Zhang, Hong; Zheng, Liancun; Si, Xinhui

Published in:

Powder Technology

DOI:

10.1016/j.powtec.2016.12.033

Publication date:

2017

Licence:

CC BY-NC-ND

Document Version

Peer reviewed version

Link to publication in Discovery Research Portal

Citation for published version (APA):

Li, L., Lin, P., Zhang, H., Zheng, L., \& Si, X. (2017). Asymptotic solutions for laminar flow based on blood circulation through a uniformlyporous channel with retractable walls and an applied transverse magnetic field. Powder Technology, 308, 398-409. https://doi.org/10.1016/j.powtec.2016.12.033

\section{General rights}

Copyright and moral rights for the publications made accessible in Discovery Research Portal are retained by the authors and/or other copyright owners and it is a condition of accessing publications that users recognise and abide by the legal requirements associated with these rights.

- Users may download and print one copy of any publication from Discovery Research Portal for the purpose of private study or research.

- You may not further distribute the material or use it for any profit-making activity or commercial gain.

- You may freely distribute the URL identifying the publication in the public portal.

Take down policy

If you believe that this document breaches copyright please contact us providing details, and we will remove access to the work immediately and investigate your claim. 


\title{
Asymptotic solutions for laminar flow based on blood circulation through a uniformly porous channel with retractable walls and an applied transverse magnetic field
}

\author{
Lin Li ${ }^{\mathrm{a}}$, Ping Lin ${ }^{\mathrm{b}}$, Hong Zhang ${ }^{\mathrm{a}}$, Liancun Zheng ${ }^{\mathrm{a}}$, Xinhui $\mathrm{Si}^{\mathrm{a}, *}$ \\ ${ }^{a}$ School of Mathematics and Physics, University of Science and Technology Beijing, Beijing, 100083, China \\ ${ }^{b}$ Division of Mathematics, University of Dundee, Dundee DD1 4HN, United Kingdom
}

\begin{abstract}
This paper is concerned with asymptotic solutions of a nonlinear boundary value problem (BVP), which arises in a study of laminar flow in a uniformly porous channel with retractable walls and an applied transverse magnetic field. For different ranges of the control parameters (i.e. $\alpha$, Re and $M)$ arising in the BVP, four cases are considered using different singular perturbation methods. For the first case, unlike those in the existing literature, we make use of the Lighthill method and successfully construct an asymptotic solution with high-order derivatives at the center of the channel. For the second case, under large suction we consider $M^{2}=O(1)$ and $M^{2}=O(R e)$, respectively, which will further extend the applying range of asymptotic solutions. In other cases, asymptotic solutions with a boundary layer are successfully constructed. In addition, numerical solutions presented for each case agree well with asymptotic solutions, which illustrates that the asymptotic solutions constructed in this paper are more reliable. Finally, the influences of some parameters on flow field are discussed to develop a better understanding of the flow problem.
\end{abstract}

Keywords: laminar flow; porous and retractable channel; magnetic field; singular perturbation method; bvp4c

\section{Introduction}

Blood circulating in the blood vessel has a strong effect on the human body and also serves as one of the basic substances constituting the human body. Its dynamics is closely associated with people's health. For example, as said by Srivastava [1], atherosclerosis, a leading cause of death in many countries, is one of the phenomenon in which the flow behavior of the blood in the vessel will be influenced by the intimal thickening of stenos artery. When severe stenosis suppresses the speed of blood, the blood supply and oxygen to the brain are reduced. Under this situation some cells in the brain start to die and then the resulting serious diseases will appear (e.g. strokes). So studies of fluid transport in the vessel can serve to better understand the functions of biological organisms (e.g. lung and cardiac).

When concerning systemic circulation in blood circulation, the blood in the left ventricle is being forced into the aorta by systole and the mitral valve between left ventricle and left atrium is closed.

${ }^{*}$ Corresponding author.Tel:+86 01062332589.

. E-mail address: sixinhui_ustb@126.com

(C) 2016. This manuscript version is made available under the CC-BY-NC-ND 4.0 license http://creativecommons.org/licenses/by-nc-nd/4.0/ 
At this juncture the left ventricle forms a vessel with one end closed. Meanwhile, the mass transfer of the vessel between inside and outside can be achieved by the seepage across permeable wall of the vessel $[2,3,4]$. Furthermore, some idealized mathematical models are proposed which consider the vessel to be permeable $[5,6]$. So studies on such flow dynamics can be meaningful in the field of bioengineering and medicine. In 1990, a mathematical model on the viscous flow of Newtonian fluid inside a permeable tube with expanding or contracting cross section was established by Goto and Uchida [7]. In their work, a expansion ratio $\alpha$ and a cross-flow Reynolds number $R e$ (defined in Section 2) were introduced to measure the expansion of the pipe and the mass transfer, respectively. Later, Dauenhauer and Majdalani [8] considered the case that laminar flow in a porous channel with expanding or contracting walls and thus established a mathematical model. So far there have existed some studies on the mathematical model. To list a few, one may count Majdalani et al. [9], Asghar et al. [10] and Hang Xu et al. [11]. On the other hand, some of medical literature have also shown that certain external factors can change the hydrodynamic in blood flow. When the blood is regarded as an electrically conducting fluid, the control of blood flow can be achieved by the application of the magnetic field (Noting that the fluid is often called as Magnetohydrodynamics or MHD). Based on the experimental investigation, Karmilov [12] has revealed that the magnetic field exerted a most significant influence on the vascular system. Subsequently, Sambasiva [13] also studied an unsteady MHD blood flow through a porous channel with porous walls. So far, some valuable results on MHD in a pipe have been reported. For example, as said in [14], the effects of MHD on blood flow are as follows: i) to reduce the high shear stress caused by stenosis and hence to prevent the damage to the red and endothelial cells, which will help bioengineers in the design of artificial organs and the treatment of vascular diseases (e.g. [15, 16, 17, 18]). ii) to delay the transition from laminar to turbulent flow inside the blood vessel and thus reducing high intensity shear zones, which are unfavorable to the blood and arterial wall. This may be vital to watch out for the symptoms of a carotid artery blockage (e.g. [19, 20]). Motivated by above works, we have realized the importance of magnetic field appearing in a model of laminar flow in a porous pipe with expanding or contracting walls. However, very little is known so far about the result of laminar flow in a porous channel with expanding or contracting walls and an applied transverse magnetic field. Therefore, based on the work [8], a principle objective of the current study is to overcome a deficiency in their model that does not account for the presence of a magnetic field. In fact, the investigation of the steady flow of an electrically conducting viscous fluid through a semi-infinite flat plate with an applied transverse magnetic field has been initiated by Suryaprakasrao [21], who obtained an asymptotic solution for small Hartmann numbers (defined in Section 2). Later, Terrill and Shrestha $[22,23]$ extended Suryaprakasrao's work by considering laminar flow in a porous channel with motionless walls and an applied transverse magnetic field. In their studies, based on either numerical or asymptotic approaches, some solutions were obtained for both small and large Reynolds numbers and all values of Hartmann number.

In fact, for the viscous flow in a porous channel with stationary walls, the earliest researcher can be traced back to Berman [24]. In his study, a nonlinear boundary value problem (BVP) with a cross-flow Reynolds number Re was obtained from the classical Navier-Stokes equations. For small $R e$, he constructed an asymptotic solution using a regular perturbation method. Subsequently, a number of further studies about the existence of multiple solutions of such a BVP followed shortly thereafter. Among these are the works of Robinson [25], Skalak and Wang [26], Shih [27], Stephen [28] and $\mathrm{Lu}[29,30,31,32,33]$. Recently, when the walls of the channel were not motionless, Hang $\mathrm{Xu}$ et al. [11] obtained three solutions for large suction using homotopy analysis method (HAM). In addition, the temporal and spatial stabilities have also considerable attention in the past due 
to the existence of multiple solutions of the BVP, where one may count Brady [34], Durlofsky and Brady [35], Sobey and Drazin [36], Zaturska, Drazin and Banks [37].

The purpose of this paper is to extend previous investigations by presenting asymptotic solutions for laminar flow in a porous channel with expanding or contracting walls and an applied transverse magnetic field. Specifically, in Section 2, by introducing the flow geometry, governing equations with boundary conditions and a stream function, a BVP (i.e. (13)-(14)) including three parameters (i.e. $\alpha, R e$ and $M$ ) is obtained. In general, when constructing a perturbation solution of the BVP, we should consider the order of magnitude among these parameters, otherwise the perturbation solution constructed is only valid for the limited scope of parameters. Therefore, Section 3 serves to present asymptotic solutions for different cases. The asymptotic and numerical solutions are compared and discussed in Section 4. Finally, Section 5 concludes the paper.

\section{Mathematical formulation of the problem}

We assume that the channel is of semi-infinite length with one closed end. In addition, to consider a two-dimensional flow, we assume that the distance $2 a$ between the porous walls is much smaller than the channel's width. Both sidewalls are assumed to have equal permeability $-v_{w}$ and to expand or contract uniformly by a time-dependent rate $\dot{a}(t)$. As shown in Fig.1, $x$ and $y$ indicate the streamwise direction and the normal direction, respectively. $u$ and $v$ denote the velocity components along $x$ - and $y$-axes. The flow velocity is zero at the closed end $(x=0)$. As a result, the motion of a viscous incompressible and electrically conducting fluid through a porous channel with an applied transverse magnetic field can be described by the following equations:

$$
\nabla \cdot \mathbf{V}=0
$$

and

$$
\frac{\partial \boldsymbol{V}}{\partial t}+(\boldsymbol{V} \cdot \nabla) \boldsymbol{V}=-\frac{1}{\rho} \nabla p+\nu \nabla^{2} \boldsymbol{V}+\frac{1}{\rho} \boldsymbol{J} \times \boldsymbol{B}
$$

where $\boldsymbol{J}$ and $\boldsymbol{B}$ are given by the Maxwell equations

$$
\begin{gathered}
\nabla \times \mathbf{H}=4 \pi \boldsymbol{J}, \\
\nabla \times \mathbf{E}=0, \\
\nabla \cdot \mathbf{B}=0,
\end{gathered}
$$

and Ohm's law

$$
\boldsymbol{J}=\sigma[\boldsymbol{E}+\boldsymbol{V} \times \boldsymbol{B}],
$$

where $\boldsymbol{B}=\mu_{m} \boldsymbol{H}, \mathbf{V}=(u, v)$ and the symbols $\nu, \sigma$, and $\mu_{m}$ represent the viscosity of the fluid, the electrical conductivity and the magnetic permeability, respectively.

For simplicity, we further assume that a constant magnetic field of strength $H_{0}$ is applied perpendicular to the walls and there is no external electric field. Meanwhile, here the induced magnetic and electric fields produced by the motion of the electrically conducting fluid are neglected. With these assumptions the magnetic term $\mathbf{J} \times \mathbf{B}$ in (2) reduces to

$$
\mathbf{J} \times \mathbf{B}=-\sigma H_{0}^{2} \mathbf{V}
$$

When we take into account the symmetry with respect to the midsection plane, the necessary 
boundary conditions for the half-domain $(y \geq 0)$ may be as follows:

$$
\begin{gathered}
u=0, \quad v=-v_{w} ; \quad y=a(t), \\
\frac{\partial u}{\partial y}=0, \quad v=0 ; \quad y=0, \\
u=0, \quad v=0 ; \quad x=0 .
\end{gathered}
$$

An appropriate stream function $\phi$ is introduced, denoting

$$
\phi=\frac{\nu x}{h} F\left(y^{*}, t\right)
$$

where $y^{*}=\frac{y}{a}$ is a dimensionless variable. When there is no confusion, we will delete the superscript $*$ in the following derivation. Then the velocity components become

$$
u=\frac{\nu x}{a^{2}} F_{y}, \quad v=-\frac{\nu}{a} F
$$

Substituting (7) and (12) into (1)-(2) and denoting $f=\frac{F}{R e}$, with the assumptions that the wall expansion ratio $\alpha$ is constant and $F$ is made dependent on $y$ and $\alpha$ (see [8] for more detail on them), we can obtain the BVP of the form

$$
f^{\prime \prime \prime}+\alpha\left(y f^{\prime \prime}+2 f^{\prime}\right)+R e\left(f f^{\prime \prime}-f^{\prime 2}\right)-M^{2} f^{\prime}=k,
$$

and the boundary conditions

$$
f(0)=0, \quad f^{\prime \prime}(0)=0, \quad f(1)=1, \quad f^{\prime}(1)=0,
$$

where $R e=\frac{a v_{w}}{\nu}$ is called as the cross-flow Reynolds number $(R e>0$ for injection and $R e<0$ for suction), $\alpha=\frac{a \dot{a}}{\nu}$ is called as the wall expansion ratio, $M=\mu_{m} H_{0} a\left(\frac{\sigma}{\rho \nu}\right)^{\frac{1}{2}}$ is called as the Hartmann number, $k$ is an integration constant and ' denotes differentiation with respect to $y$.

\section{Asymptotic solutions for the BVP (i.e. (13) and (14))}

The main aim of this section is to present asymptotic solutions for these parameters (i.e. $\alpha$, $R e$ and $M$ ) with different orders of magnitude. To show them more clearly, the contents on them will be separated in Sections 3.1-3.4, respectively.

\subsection{Solution for large injection Reynolds numbers}

For large injection, we consider the case $\alpha=O(1)$ and $M^{2}=O(1)$, and treat $\varepsilon=\frac{1}{R e}$ as the perturbation parameter. Then Eq.(13) becomes

$$
\varepsilon f^{\prime \prime \prime}+\varepsilon \alpha\left(y f^{\prime \prime}+2 f^{\prime}\right)+\left(f f^{\prime \prime}-f^{\prime 2}\right)-\varepsilon M^{2} f^{\prime}=\varepsilon k .
$$

Before constructing the asymptotic solution, we first introduce a result in [38]. When $M=0$, Majdalani and Zhou have solved Eq.(13) asymptotically using a regular perturbation method and 
the variation of parameters method. The corresponding asymptotic solution is as follows:

$$
\begin{aligned}
f(\varsigma)= & \sin \varsigma+\varepsilon\left\{-\frac{2 \alpha}{\pi} \varsigma+\left(\frac{\pi}{4}-\frac{4 \alpha}{\pi}\right) \times\left[(\varsigma \cos \varsigma-\sin \varsigma) \ln \tan \frac{1}{2} \varsigma-\cos \varsigma S(\varsigma)\right]+\alpha \sin \varsigma+\right. \\
& {\left.\left[\left(\frac{1}{2}-8 \alpha \pi^{-2}\right) S\left(\frac{1}{2} \pi\right)+4 \alpha \pi^{-2}-\frac{1}{2}\right] \varsigma \cos \varsigma\right\}, }
\end{aligned}
$$

where $\varsigma=\frac{1}{2} \pi y$ and $S(\varsigma)=\int_{0}^{\varsigma} \phi \csc \phi d \phi$. When (16) is differentiated three times, we can obtain

$$
\begin{aligned}
f^{\prime \prime \prime}(\varsigma)= & \frac{1}{8} \pi^{3}\left(-\cos \varsigma+\varepsilon\left\{-\alpha \cos \varsigma+\left(\frac{1}{4} \pi-\frac{4 \alpha}{\pi}\right) \times\left[-\sin \varsigma S(\varsigma)-(2 \cos \varsigma-\varsigma \sin \varsigma) \ln \tan \frac{1}{2} \varsigma-1\right]\right.\right. \\
& \left.\left.-\left[\left(\frac{1}{2}-8 \alpha \pi^{-2}\right) S\left(\frac{1}{2} \pi\right)+4 \alpha \pi^{-2}-\frac{1}{2}\right](3 \cos \varsigma-\varsigma \sin \varsigma)\right\}\right)
\end{aligned}
$$

where' denotes differentiation with respect to $\varsigma$. As observed (17), it becomes unbounded as $\varsigma \rightarrow 0$ due to the secular term $\cos \varsigma \ln \tan \frac{1}{2} \varsigma$. Besides this, when $\alpha=0$, the series solution, given by Yuan [39], also exhibits the similar feature (i.e. the third derivative of the series solution tends to infinite at the center of the channel). However, here the unboundedness does not seem to occur in practical application. Later, the existence of a viscous shear layer, pointed out by Terrill [40], would result in the appearance of the unboundedness. To eliminate the unboundedness in $f^{\prime \prime \prime}$, Zhou and Majdalani [38] obtained a uniformly valid composite solution using matched-asymptotic expansions with logarithmic corrections. However, in the current study, we will not plan to follow their line due to the complexity of matching process. The Lighthill method (Noting that it is a method of strained coordinates, the reader can see [41] for more detail on it) is used to eliminate the secular term. The specific process is as follows: Firstly, we introduce a variable transformation on $y$ :

$$
y=\xi+\varepsilon X_{1}(\xi)+\varepsilon^{2} X_{2}(\xi)+O\left(\varepsilon^{3}\right),
$$

where the functions $X_{1}, X_{2}$ will be determined later. $f$ and $\varepsilon k$ can be expanded as the following forms:

$$
\begin{gathered}
f(y) \triangleq g(\xi)=g_{0}(\xi)+\varepsilon g_{1}(\xi)+\varepsilon^{2} g_{2}(\xi)+O\left(\varepsilon^{3}\right), \\
\varepsilon k=\lambda_{0}+\varepsilon \lambda_{1}+\varepsilon^{2} \lambda_{2}+O\left(\varepsilon^{3}\right) .
\end{gathered}
$$

Substituting (18)-(20) into (15) and equating coefficients of $\varepsilon^{n}$, one can obtain

$$
\begin{gathered}
\varepsilon^{0}: g_{0} \ddot{g}_{0}-\dot{g}_{0}{ }^{2}=\lambda_{0} \\
\varepsilon: \dddot{g}_{0}+\alpha\left(\xi \ddot{g}_{0}+2 \dot{g}_{0}\right)+g_{0} \ddot{g}_{1}+g_{1} \ddot{g}_{0}-2 \dot{g}_{0} \dot{g}_{1}-M^{2} \dot{g}_{0}=\lambda_{1}+3 \dot{X}_{1} \lambda_{0} \\
\ldots \ldots .
\end{gathered}
$$

Here - denotes the derivative with respect to $\xi$. According to (14), the boundary conditions corresponding to $g_{i}(i=0,1,2, \cdots)$ can be induced: 


\section{i). The wall of the channel (i.e. $y=1$ )}

We assume that $\widetilde{\xi}$ is the root of (18) at $y=1$, then

$$
\begin{aligned}
\widetilde{\xi} & =1-\varepsilon X_{1}(\widetilde{\xi})-\varepsilon^{2} X_{2}(\widetilde{\xi})+O\left(\varepsilon^{3}\right) \\
& =1-\varepsilon\left\{X_{1}(1)+\dot{X}_{1}(1)\left[-\varepsilon X_{1}(\widetilde{\xi})-\varepsilon^{2} X_{2}(\widetilde{\xi})\right]+\cdots\right\}-\varepsilon^{2}\left\{X_{2}(1)+\dot{X}_{2}(1)\left[-\varepsilon X_{1}(\widetilde{\xi})-\varepsilon^{2} X_{2}(\widetilde{\xi})\right]+\cdots\right\} \\
& \cdots \cdots \\
& =1-\varepsilon X_{1}(1)-\varepsilon^{2}\left[X_{2}(1)-\dot{X}_{1}(1) X_{1}(1)\right]+O\left(\varepsilon^{3}\right) .
\end{aligned}
$$

Using (23), we can induce

$$
\begin{aligned}
\left.f\right|_{y=1}=1 \Longrightarrow 1 & =\left.g\right|_{\xi=\widetilde{\xi}}=\left.g\right|_{\xi=1}+\left.\dot{g}\right|_{\xi=1}\left\{-\varepsilon X_{1}(1)-\varepsilon^{2}\left[X_{2}(1)-\dot{X}_{1}(1) X_{1}(1)\right]+\cdots\right\} \\
& =\left.g_{0}\right|_{\xi=1}+\left.\varepsilon\left[g_{1}-X_{1} \dot{g}_{0}\right]\right|_{\xi=1}+O\left(\varepsilon^{2}\right), \\
\left.f^{\prime}\right|_{y=1}=0 \Longrightarrow 0 & =\left.\dot{g}\right|_{\xi=\widetilde{\xi}}=\left.\dot{g}\right|_{\xi=1}+\left.\ddot{g}\right|_{\xi=1}\left\{-\varepsilon X_{1}(1)-\varepsilon^{2}\left[X_{2}(1)-\dot{X}_{1}(1) X_{1}(1)\right]+\cdots\right\} \\
& =\left.\dot{g}_{0}\right|_{\xi=1}+\left.\varepsilon\left[\dot{g}_{1}-X_{1} \ddot{g}_{0}\right]\right|_{\xi=1}+O\left(\varepsilon^{2}\right) .
\end{aligned}
$$

The resulting boundary conditions at $y=1$ become

$$
\begin{gathered}
\left.g_{0}\right|_{\xi=1}=1, \quad g_{1}-\left.X_{1} \dot{g}_{0}\right|_{\xi=1}=0, \cdots \\
\left.\dot{g}_{0}\right|_{\xi=1}=0, \quad \dot{g}_{1}-\left.X_{1} \ddot{g}_{0}\right|_{\xi=1}=0, \cdots .
\end{gathered}
$$

ii). The center of the channel (i.e. $y=0$ )

We suppose that $\widehat{\xi}$ is the root of (18) at $y=0$, then

$$
\begin{aligned}
\widehat{\xi}=-\varepsilon X_{1}(\widehat{\xi}) & \left.\left.-\varepsilon^{2} X_{2}(\widehat{\xi})\right)+O\left(\varepsilon^{3}\right)=-\varepsilon X_{1}(0)-\varepsilon^{2}\left[X_{2}(0)-\dot{X}_{1}(0) X_{1}(0)\right)\right]+O\left(\varepsilon^{3}\right) \\
\left.f\right|_{y=0}=0 \Longrightarrow 0 & =\left.g\right|_{\xi=\widehat{\xi}}=\left.g\right|_{\xi=0}+\left.\dot{g}\right|_{\xi=0}\left\{-\varepsilon X_{1}(0)-\varepsilon^{2}\left[X_{2}(0)-\dot{X}_{1}(0) X_{1}(0)\right]+\cdots\right\} \\
& =\left.g_{0}\right|_{\xi=0}+\left.\varepsilon\left[g_{1}-X_{1} \dot{g}_{0}\right]\right|_{\xi=0}+O\left(\varepsilon^{2}\right) \\
\left.f^{\prime \prime}\right|_{y=0}=0 \Longrightarrow 0 & =\ddot{g}+\left.\dot{g}\left[\frac{-\varepsilon \ddot{X}_{1}-\varepsilon^{2} \ddot{X}_{2}}{1+\varepsilon \dot{X}_{1}+\varepsilon^{2} \dot{X}_{2}}\right]\right|_{\xi=\widehat{\xi}} \\
& =\left\{\left.\ddot{g}\right|_{\xi=0}+\left.\dddot{g}\right|_{\xi=0}\left[-\varepsilon X_{1}(0)-\varepsilon^{2}\left(X_{2}(0)-\dot{X}_{1}(0) X_{1}(0)\right)+\cdots\right]\right\}+\left\{\left.\dot{g}\right|_{\xi=0}\right. \\
& \left.+\left.\ddot{g}\right|_{\xi=0}\left[-\varepsilon X_{1}(0)-\varepsilon^{2}\left(X_{2}(0)-\dot{X}_{1}(0) X_{1}(0)\right)+\cdots\right]\right\}\left.\cdot\left[\frac{-\varepsilon \ddot{X}_{1}-\varepsilon^{2} \ddot{X}_{2}}{1+\varepsilon \dot{X}_{1}+\varepsilon^{2} \dot{X}_{2}}\right]\right|_{\xi=\widehat{\xi}} \\
& =\left.\ddot{g}_{0}\right|_{\xi=0}+\left.\varepsilon\left[\ddot{g}_{1}-X_{1} \dddot{g}_{0}-\ddot{X}_{1} \dot{g}_{0}\right]\right|_{\xi=0}+O\left(\varepsilon^{2}\right) .
\end{aligned}
$$

The boundary conditions at $y=0$ become

$$
\begin{gathered}
\left.g_{0}\right|_{\xi=0}=0, \quad g_{1}-\left.X_{1} \dot{g}_{0}\right|_{\xi=0}=0, \cdots \\
\left.\ddot{g}_{0}\right|_{\xi=0}=0, \quad \ddot{g}_{1}-X_{1} \dddot{g}_{0}-\left.\ddot{X}_{1} \dot{g}_{0}\right|_{\xi=0}=0, \cdots .
\end{gathered}
$$


Using (26)-(27) and (31)-(32), the solution for (21) can be obtained as follows:

$$
g_{0}=\sin \left(\frac{\pi}{2} \xi\right) \triangleq \sin \theta
$$

where $\theta=\frac{\pi}{2} \xi$ and $\lambda_{0}=-\frac{\pi^{2}}{4}$. Substituting (33) into (22) yields the equation for $g_{1}$ :

$$
\sin \theta g_{1}^{\prime \prime}(\theta)-2 \cos \theta g_{1}^{\prime}(\theta)-\sin \theta g_{1}(\theta)=\left(\frac{\pi}{2}-\frac{4 \alpha}{\pi}+\frac{2 M^{2}}{\pi}\right) \cos \theta-2 \dot{X}_{1}(\xi)+\frac{2 \alpha}{\pi} \theta \sin \theta+\frac{4}{\pi^{2}} \lambda_{1},
$$

where ' denotes the derivative with respect to $\theta$. To eliminate the singularity that may appear in $g_{1}$, we can set

$$
\left(\frac{\pi}{2}-\frac{4 \alpha}{\pi}+\frac{2 M^{2}}{\pi}\right) \cos \theta-2 \dot{X}_{1}(\xi)+\frac{2 \alpha}{\pi} \theta \sin \theta+\frac{4}{\pi^{2}} \lambda_{1}=0 .
$$

Obviously, from (35), one can obtain

$$
X_{1}(\theta)=\left(\frac{1}{2}-\frac{2 \alpha}{\pi^{2}}+\frac{2 M^{2}}{\pi^{2}}\right) \sin \theta-\frac{2 \alpha}{\pi^{2}} \theta \cos \theta+\frac{4 \lambda_{1}}{\pi^{3}} \theta+C_{1},
$$

where $C_{1}$ is an integration constant. At this juncture, Eq.(34) becomes

$$
\sin \theta g_{1}^{\prime \prime}(\theta)-2 \cos \theta g_{1}^{\prime}(\theta)-\sin \theta g_{1}(\theta)=0
$$

According to [9], for the general homogeneous equation (37), its solution can be shown as follows:

$$
g_{1}(\theta)=K_{1} \cos \theta+K_{2}(\sin \theta-\theta \cos \theta),
$$

where $K_{1}$ and $K_{2}$ are integration constants. Applying boundary conditions (26)-(27) and (31)-(32), one obtains

$$
K_{1}=\frac{\pi}{2} C_{1}, \quad K_{2}=0 .
$$

When considering the non-zero solution of $g_{1}$ and setting $C_{1}=1$, we can obtain

$$
g_{1}=\frac{\pi}{2} \cos \theta, \quad \lambda_{1}=-\frac{\pi^{2}}{4}+\alpha-M^{2} .
$$

Finally, by combining (40) with (33), the resulting solution $f(y)$ becomes as follows:

$$
f(y)=\sin \left(\frac{\pi}{2} \xi\right)+\varepsilon \frac{\pi}{2} \cos \left(\frac{\pi}{2} \xi\right)
$$

where $y=\xi+\varepsilon\left[\left(\frac{1}{2}-\frac{2 \alpha}{\pi^{2}}+\frac{2 M^{2}}{\pi^{2}}\right) \sin \left(\frac{\pi}{2} \xi\right)-\frac{\alpha}{\pi} \xi \cos \left(\frac{\pi}{2} \xi\right)+\left(\frac{1}{2}-\frac{2 \alpha}{\pi^{2}}+\frac{2 M^{2}}{\pi^{2}}\right) \xi+1\right]$.

To verify the validity of the asymptotic solution (41), Table 1 not only presents the comparison between asymptotic and numerical solutions ( $M e e=2 ; \alpha= \pm 2$ ), but also shows the comparison our results with the analytical solution obtained by Majdalani et al. [9] for $\alpha=2, M=0$. As observed from Table 1, the last three columns indicate that our asymptotic results are closer to numerical results in comparison to ones obtained by Majdalani et al. In a sense, this also illustrates that applying the Lighthill method is reliable for large injection. Therefore, we hope that it can be extended to the similar flow problems. 


\subsection{Solution for large suction Reynolds numbers}

For large suction, under $M=0$ Majdalani et al. $[8,9]$ have pointed out the existence of thinning boundary layer. Here we not only consider the boundary layer, but also further extend to the case $M^{2}=O(R e)$. For added clarity, this section is divided into two parts: the first part$\alpha=O(1)$ and $M^{2}=O(1)$ as $R e \rightarrow-\infty$, and the second part $-\alpha=O(1)$ and $M^{2}=O(R e)$ as $R e \rightarrow-\infty$.

\section{A. $\alpha=O(1)$ and $M^{2}=O(1)$ as $R e \rightarrow-\infty$}

As mentioned before, a viscous boundary layer is formed near the walls of the channel. To better describe it, the method of boundary layer correction (Noting that the reader can see [42, 43, 44] for more detail on this method) is used to deal with it. The specific process is as follows:

We define $\varepsilon=\frac{1}{R e}$ to be our perturbation parameter, and set $f^{\prime}(0)=\beta$ and $f^{\prime \prime \prime}(0)=\delta$, then Eq.(13) can be written as

$$
\varepsilon f^{\prime \prime \prime}+\varepsilon \alpha\left(y f^{\prime \prime}+2 f^{\prime}\right)+\left(f f^{\prime \prime}-f^{\prime 2}\right)-\varepsilon M^{2} f^{\prime}=\varepsilon \delta+2 \varepsilon \alpha \beta-\beta^{2}-\varepsilon M^{2} \beta,
$$

where $\varepsilon k=\varepsilon \delta+2 \varepsilon \alpha \beta-\beta^{2}-\varepsilon M^{2} \beta$.

We introduce a variable transformation of the form

$$
1-y=\varepsilon \tau
$$

and expand the function $f$ into a composite form as follows:

$$
f(y)=f_{0}(y)+\varepsilon\left(f_{1}(y)+g_{1}(\xi)\right)+\varepsilon^{2}\left(f_{2}(y)+g_{2}(\xi)\right)+\varepsilon^{3}\left(f_{3}(y)+g_{3}(\xi)\right)+O\left(\varepsilon^{4}\right) .
$$

Noting that $g_{i}(\xi), i=1,2, \cdots$, are boundary layer functions and rapidly decay when $y$ is away from the walls. In addition, the constants $\beta, \delta$ can be written as follows:

$$
\left.\begin{array}{c}
\beta=\beta_{0}+\varepsilon \beta_{1}+\varepsilon^{2} \beta_{2}+\varepsilon^{3} \beta_{3}+O\left(\varepsilon^{4}\right) \\
\delta=\delta_{0}+\varepsilon \delta_{1}+\varepsilon^{2} \delta_{2}+\varepsilon^{3} \delta_{3}+O\left(\varepsilon^{4}\right)
\end{array}\right\} .
$$

Using (43)-(45), the boundary conditions (14) become

$$
\begin{aligned}
f(0)=0, f^{\prime \prime}(0) & =\left.0 \Longrightarrow f_{i}\right|_{y=0}=0,\left.f_{i}^{\prime \prime}\right|_{y=0}=0, i=0,1,2, \cdots \\
f(1) & =\left.1 \Longrightarrow f_{0}\right|_{y=1}=1,\left.f_{i}\right|_{y=1}+\left.g_{i}\right|_{\tau=0}=0, i=1,2, \cdots \\
f^{\prime}(1) & =\left.0 \Longrightarrow f_{i}^{\prime}\right|_{y=1}-\left.\dot{g}_{i+1}\right|_{\tau=0}=0, i=0,1,2, \cdots
\end{aligned}
$$

Here' and - denote the derivatives with respect to $y$ and $\tau$, respectively. Substituting (44)-(45) into (42) and equating coefficients of $\varepsilon^{n}$, one can obtain

$$
f_{0} f_{0}^{\prime \prime}-f_{0}^{\prime 2}=-\beta_{0}^{2} .
$$

The corresponding boundary conditions become

$$
\left.f_{0}\right|_{y=0}=0,\left.\quad f_{0}^{\prime \prime}\right|_{y=0}=0,\left.\quad f_{0}\right|_{y=1}=1 .
$$


As a result, we have

$$
f_{0}=y, \quad \beta_{0}=1
$$

For $g_{1}$, the equation becomes

$$
-\dddot{g}_{1}+\ddot{g}_{1}=0,
$$

and the corresponding boundary condition becomes

$$
\left.\dot{g}_{1}\right|_{\tau=0}=\left.f_{0}^{\prime}\right|_{y=1}=1 .
$$

According to the character of the boundary function, the expression of $g_{1}$ should be

$$
g_{1}=e^{\tau} .
$$

Next, we have the following equation:

$$
y f_{1}^{\prime \prime}-2 f_{1}^{\prime}=-2 \beta_{1},
$$

and the corresponding boundary conditions are as follows:

$$
\left.f_{1}\right|_{y=0}=0,\left.\quad f_{1}^{\prime \prime}\right|_{y=0}=0,\left.\quad f_{1}\right|_{y=1}=-\left.g_{1}\right|_{\tau=0}=-1 .
$$

From (55)-(56), we can obtain the following results:

$$
f_{1}=-y, \quad \beta_{1}=-1 .
$$

At this juncture, for $g_{2}$, the boundary condition becomes

$$
\left.\dot{g}_{2}\right|_{\tau=0}=\left.f_{1}^{\prime}\right|_{y=1}=-1 .
$$

On the other hand, the corresponding equation becomes

$$
-\dddot{g}_{2}+\ddot{g}_{2}+\alpha e^{\tau}+e^{\tau}-\tau e^{\tau}=0 .
$$

So we can obtain

$$
g_{2}=e^{\tau}\left[-\frac{1}{2} \tau^{2}+(\alpha+3) \tau-(\alpha+4)\right] .
$$

Following the process above, we can easily obtain the following results:

$$
\begin{gathered}
f_{2}=(\alpha+4) y \\
f_{3}=\left(-\alpha^{2}-11 \alpha+M^{2}-\frac{129}{4}\right) y, \\
g_{3}=-\frac{1}{4} e^{2 \tau}+e^{\tau}\left[\frac{1}{8} \tau^{4}-\frac{1}{2}(\alpha+3) \tau^{3}+\left(\frac{1}{2} \alpha^{2}+3 \alpha+9\right) \tau^{2}+\left(-\alpha^{2}-10 \alpha+M^{2}-28\right) \tau+\right. \\
\left.\alpha^{2}+11 \alpha-M^{2}+\frac{65}{2}\right] .
\end{gathered}
$$


Finally, the composite solution $f$ becomes as follows:

$$
\begin{aligned}
f & =y+\varepsilon\left\{-y+e^{\tau}\right\}+\varepsilon^{2}\left\{(\alpha+4) y+e^{\tau}\left[-\frac{1}{2} \tau^{2}+(\alpha+3) \tau-(\alpha+4)\right]\right\}+\varepsilon^{3}\left\{\left(-\alpha^{2}-11 \alpha\right.\right. \\
& \left.+M^{2}-\frac{129}{4}\right) y-\frac{1}{4} e^{2 \tau}+e^{\tau}\left[\frac{1}{8} \tau^{4}-\frac{1}{2}(\alpha+3) \tau^{3}+\left(\frac{1}{2} \alpha^{2}+3 \alpha+9\right) \tau^{2}+\left(-\alpha^{2}-10 \alpha+M^{2}\right.\right. \\
& \left.\left.-28) \tau+\alpha^{2}+11 \alpha-M^{2}+\frac{65}{2}\right]\right\} .
\end{aligned}
$$

A comparison of $-f^{\prime \prime}(1)$ between our results and Majdalani et al. [9] is presented in Table 2. As observed, we can find that the biggest error occurs in the case $\alpha=-20$ and $M=52$. This is because $M^{2}$ is too large to meet the assumption $M^{2}=O(1)$. So, in the next section we will consider the case $M^{2}=O(R e)$.

\section{B. $\alpha=O(1)$ and $M^{2}=O(R e)$ as $R e \rightarrow-\infty$}

Since $M^{2}=O(R e)$ and $r=-\frac{M^{2}}{R e}$, we can derive $r \sim O(1)$. In addition, we denote $\varepsilon=\frac{1}{R e}$, $f^{\prime}(0)=\beta$, and $f^{\prime \prime \prime}(0)=\delta$. Eq. $(13)$ can be written as

$$
\varepsilon f^{\prime \prime \prime}+\varepsilon \alpha\left(y f^{\prime \prime}+2 f^{\prime}\right)+\left(f f^{\prime \prime}-f^{\prime 2}\right)+r f^{\prime}=\varepsilon \delta+2 \varepsilon \alpha \beta-\beta^{2}+r \beta,
$$

where $\varepsilon k=\varepsilon \delta+2 \varepsilon \alpha \beta-\beta^{2}+r \beta$. Following the procedure in Part A, we can induce the expressions for $f_{i}$ and $g_{i}$ as follows:

$$
\begin{gathered}
f_{0}=y, \\
f_{1}=-y, \\
f_{2}=(\alpha+4-r) y, \\
f_{3}=\left(-\alpha^{2}-11 \alpha+3 \alpha r-2 r^{2}+15 r-\frac{129}{4}\right) y, \\
g_{1}=e^{\tau}, \\
g_{2}=e^{\tau}\left[-\frac{1}{2} \tau^{2}+(\alpha+3-r) \tau-(r-\alpha-4)\right], \\
-\frac{1}{4} e^{2 \tau}+e^{\tau}\left[\frac{1}{8} \tau^{4}+\frac{1}{2}(r-\alpha-3) \tau^{3}+\left(\frac{1}{2} \alpha^{2}+3 \alpha-\alpha r+\frac{1}{2} r^{2}-4 r+9\right) \tau^{2}+\left(-\alpha^{2}\right.\right. \\
\left.\left.-10 \alpha+3 \alpha r-2 r^{2}+14 r-28\right) \tau+\alpha^{2}+11 \alpha-3 \alpha r+2 r^{2}-15 r+\frac{65}{2}\right] .
\end{gathered}
$$

As a result, the composite solution of (65) becomes as follows:

$$
\begin{aligned}
f=y & +\varepsilon\left\{-y+e^{\tau}\right\}+\varepsilon^{2}\left\{(\alpha+4-r) y+e^{\tau}\left[-\frac{1}{2} \tau^{2}+(\alpha+3-r) \tau-(r-\alpha-4)\right]\right\}+\varepsilon^{3}\left\{\left(-\alpha^{2}\right.\right. \\
& \left.-11 \alpha+3 \alpha r-2 r^{2}+15 r-\frac{129}{4}\right) y-\frac{1}{4} e^{2 \tau}+e^{\tau}\left[\frac{1}{8} \tau^{4}+\frac{1}{2}(r-\alpha-3) \tau^{3}+\left(\frac{1}{2} \alpha^{2}+3 \alpha-\alpha r\right.\right. \\
& \left.+\frac{1}{2} r^{2}-4 r+9\right) \tau^{2}+\left(-\alpha^{2}-10 \alpha+3 \alpha r-2 r^{2}+14 r-28\right) \tau+\left(\alpha^{2}+11 \alpha-3 \alpha r+2 r^{2}\right. \\
& \left.\left.\left.-15 r+\frac{65}{2}\right)\right]\right\} .
\end{aligned}
$$


When comparing (73) with (64), we can find that the Hartmann number $M$ initially appears in the term $O\left(\varepsilon^{2}\right)$ for this case, which indicates the increase of the magnetic field's effect on the solution. We can observe that the asymptotic solution (73) becomes more accurate for $\alpha=-20$ and $M=52$ (see Tables 2-3).

\subsection{Solution for large Hartmann numbers}

In this section, we consider the case that the intensity of the magnetic field is large enough so that the magnetic field force is the main influence factor.

We treat $\varepsilon=\frac{1}{M^{2}}$ as the perturbation parameter and denote $\beta=f^{\prime}(0)$ and $\delta=f^{\prime \prime \prime}(0)$, then Eq.(13) can be written as

$$
\varepsilon f^{\prime \prime \prime}+\varepsilon \alpha\left(y f^{\prime \prime}+2 f^{\prime}\right)+\varepsilon R e\left(f f^{\prime \prime}-f^{\prime 2}\right)-f^{\prime}=\varepsilon \delta+2 \varepsilon \alpha \beta-\varepsilon R e \beta^{2}-\beta,
$$

where $\varepsilon k=\varepsilon \delta+2 \varepsilon \alpha \beta-\varepsilon \operatorname{Re} \beta^{2}-\beta$.

\section{A. Inner solution}

As said by Terrill et al. [22, 23], when $\alpha=0$, large Hartmann number $M$ would result in the appearance of a boundary layer near the wall. To obtain a solution within the boundary layer, an appropriate stretching transformation is introduced as follows:

$$
\begin{gathered}
\xi=\frac{1-y}{\varepsilon^{a}}, \\
f(y)=1+\varepsilon^{b} g(\xi),
\end{gathered}
$$

where $a$ and $b$ are constants that will be determined later. Substituting (75)-(76) into (74) yields $-\varepsilon^{1+b-3 a} \dddot{g}+\varepsilon^{1+b-2 a} A \ddot{g}-\varepsilon^{1+b-a} \alpha(\xi \ddot{g}+2 \dot{g})+\varepsilon^{1+2 b-2 a} \operatorname{Re}\left(g \ddot{g}-\dot{g}^{2}\right)+\varepsilon^{b-a} \dot{g}=\varepsilon \delta+2 \varepsilon \alpha \beta-\varepsilon R e \beta^{2}-\beta$,

where denotes the derivative with respect to $\xi$ and $A=\alpha+R e$.

As expected, the magnetic boundary layer involves a balance between viscous and magnetic terms. Thus we set $1+b-3 a=b-a$, which indicates $a=\frac{1}{2}$. Besides, because of the balance of the order of magnitude on both sides of Eq.(77), we have $b=a=\frac{1}{2}$. At this juncture, Eq.(77) becomes

$$
-\dddot{g}+\varepsilon^{\frac{1}{2}} A \ddot{g}-\varepsilon \alpha(\xi \ddot{g}+2 \dot{g})+\varepsilon R e\left(g \ddot{g}-\dot{g}^{2}\right)+\dot{g}=\varepsilon \delta+2 \varepsilon \alpha \beta-\varepsilon R e \beta^{2}-\beta .
$$

$g(\xi), \beta$ and $\delta$ are expanded as follows:

$$
\left.\begin{array}{rl}
g(\xi) & =g_{0}(\xi)+\varepsilon^{\frac{1}{2}} g_{1}(\xi)+\varepsilon g_{2}(\xi)+\varepsilon^{\frac{3}{2}} g_{3}+O\left(\varepsilon^{2}\right), \\
\beta & =\beta_{0}+\varepsilon^{\frac{1}{2}} \beta_{1}+\varepsilon \beta_{2}+\varepsilon^{\frac{3}{2}} \beta_{3}+O\left(\varepsilon^{2}\right), \\
\delta & =\delta_{0}+\varepsilon^{\frac{1}{2}} \delta_{1}+\varepsilon \delta_{2}+\varepsilon^{\frac{3}{2}} \delta_{3}+O\left(\varepsilon^{2}\right) .
\end{array}\right\}
$$

Substituting (79) into (78) and equating coefficients of $\varepsilon^{i}$, one can obtain

$$
\begin{gathered}
\varepsilon^{0}:-\dddot{g}_{0}+\dot{g}_{0}=-\beta_{0}, \\
\varepsilon^{\frac{1}{2}}:-\ddot{g}_{1}+\dot{g}_{1}+(\alpha+R e) \ddot{g}_{0}=-\beta_{1}, \\
\varepsilon:-\dddot{g}_{2}+\dot{g}_{2}+(\alpha+R e) \ddot{g}_{1}-\alpha\left(\xi \ddot{g}_{0}+2 \dot{g}_{0}\right)+\operatorname{Re}\left(g_{0} \ddot{g}_{0}-\dot{g}_{0}^{2}\right)=\delta_{0}+2 \alpha \beta_{0}-\operatorname{Re} \beta_{0}^{2}-\beta_{2},
\end{gathered}
$$




$$
\varepsilon^{\frac{3}{2}}:-\dddot{g}_{3}+\dot{g}_{3}+(\alpha+R e) \ddot{g}_{2}-\alpha\left(\xi \ddot{g}_{1}+2 \dot{g}_{1}\right)+\operatorname{Re}\left(g_{0} \ddot{g}_{1}+g_{1} \ddot{g}_{0}-2 \dot{g}_{0} \dot{g}_{1}\right)=\delta_{1}+2 \alpha \beta_{1}-2 R e \beta_{0} \beta_{1}-\beta_{3},
$$

The boundary conditions for $g_{n}$ become

$$
g_{n}(0)=0, \dot{g}_{n}(0)=0 ; n=0,1,2, \cdots .
$$

The solution of Eq.(80), subject to the boundary conditions in (84), is

$$
g_{0}=-\beta_{0} e^{-\xi}-\beta_{0} \xi+\beta_{0} .
$$

Using (85), the solution of Eq.(81) satisfying the boundary conditions in (84) is

$$
g_{1}=e^{-\xi}\left(-\frac{1}{2} A \beta_{0} \xi-\frac{1}{2} A \beta_{0}-\beta_{1}\right)-\beta_{1} \xi+\left(\beta_{1}+\frac{1}{2} A \beta_{0}\right) .
$$

Furthermore, the expressions of $g_{2}$ and $g_{3}$ become

$$
\begin{aligned}
g_{2}= & e^{-\xi}\left[\left(\frac{1}{4} \alpha \beta_{0}-\frac{1}{8} A^{2} \beta_{0}+\frac{1}{4} R e \beta_{0}^{2}\right) \xi^{2}+\left(-\frac{1}{4} \alpha \beta_{0}-\frac{1}{8} A^{2} \beta_{0}-\frac{1}{2} A \beta_{1}+\frac{5}{4} \operatorname{Re} \beta_{0}^{2}\right) \xi+\frac{5}{4} \operatorname{Re} \beta_{0}^{2}-\frac{1}{2} A \beta_{1}\right. \\
& \left.-\frac{1}{4} \alpha \beta_{0}-\frac{1}{8} A^{2} \beta_{0}+\delta_{0}-\beta_{2}\right]+\left(\delta_{0}-\beta_{2}\right) \xi+\left(\frac{1}{4} \alpha \beta_{0}-\frac{5}{4} \operatorname{Re} \beta_{0}^{2}+\frac{1}{2} A \beta_{1}-\delta_{0}+\beta_{2}+\frac{1}{8} A^{2} \beta_{0}\right), \\
g_{3}= & e^{-\xi}\left[\left(\frac{1}{8} A \alpha \beta_{0}+\frac{1}{8} A R e \beta_{0}^{2}-\frac{1}{48} A^{3} \beta_{0}\right) \xi^{3}+\left(-\frac{1}{8} A^{2} \beta_{1}+\frac{1}{4} \alpha \beta_{1}+\frac{1}{2} \operatorname{Re} \beta_{0} \beta_{1}+\frac{5}{8} A R e \beta_{0}^{2}-\frac{1}{8} A \alpha \beta_{0}\right) \xi^{2}\right. \\
& +\left(-\frac{1}{4} \alpha \beta_{1}-\frac{3}{8} A \alpha \beta_{0}-\frac{1}{2} A \beta_{2}+\frac{5}{2} \operatorname{Re} \beta_{0} \beta_{1}-\frac{1}{8} A^{2} \beta_{1}+\frac{1}{2} A \delta_{0}+\frac{9}{8} A R e \beta_{0}^{2}\right) \xi+\left(-\beta_{3}+\delta_{1}+\right. \\
& \left.\left.\frac{5}{2} \operatorname{Re} \beta_{0} \beta_{1}-\frac{3}{8} A \alpha \beta_{0}+\frac{1}{2} A \delta_{0}-\frac{1}{4} \alpha \beta_{1}-\frac{1}{8} A^{2} \beta_{1}+\frac{9}{8} A \operatorname{Re} \beta_{0}^{2}-\frac{1}{2} A \beta_{2}\right)\right]+\left(-\beta_{3}+\delta_{1}\right) \xi+\left(\beta_{3}-\delta_{1}\right. \\
& \left.+\frac{3}{8} A \alpha \beta_{0}+\frac{1}{4} \alpha \beta_{1}+\frac{1}{8} A^{2} \beta_{1}-\frac{1}{2} A \delta_{0}-\frac{9}{8} A R e \beta_{0}^{2}+\frac{1}{2} A \beta_{2}-\frac{5}{2} \operatorname{Re} \beta_{0} \beta_{1}\right) .
\end{aligned}
$$

Hence, the inner perturbation solution of (74) can be expressed as

$$
f^{(i)}=1+\varepsilon^{\frac{1}{2}} g_{0}+\varepsilon g_{1}+\varepsilon^{\frac{3}{2}} g_{2}+\varepsilon^{2} g_{3}+O\left(\varepsilon^{\frac{5}{2}}\right),
$$

where the coefficients $\beta_{n}$ and $\delta_{n}(n=0,1, \cdots)$ are to be determined by the matching process in Part C.

\section{B. Outer solution}

The outer solution of (74) satisfies the inviscid equation

$$
f^{\prime}=\beta,
$$

and the corresponding outer boundary conditions become

$$
f(0)=0, f^{\prime \prime}(0)=0 .
$$


Thus the outer solution $f^{(o)}(y)$ is

$$
f^{(o)}(y)=\beta y
$$

where $\beta=\beta_{0}+\varepsilon^{\frac{1}{2}} \beta_{1}+\varepsilon \beta_{2}+\varepsilon^{\frac{3}{2}} \beta_{3}+\varepsilon^{2} \beta_{4}+O\left(\varepsilon^{\frac{5}{2}}\right)$.

\section{Matching process}

If (92) is written in terms of the inner variable $\xi$, then

$$
f^{(o) i}=\left(\beta_{0}+\varepsilon^{\frac{1}{2}} \beta_{1}+\varepsilon \beta_{2}+\varepsilon^{\frac{3}{2}} \beta_{3}+\varepsilon^{2} \beta_{4}\right)-\varepsilon^{\frac{1}{2}}\left(\beta_{0}+\varepsilon^{\frac{1}{2}} \beta_{1}+\varepsilon \beta_{2}+\varepsilon^{\frac{3}{2}} \beta_{3}\right) \xi+\cdots .
$$

When $\xi \rightarrow \infty$, we can obtain

$$
\begin{aligned}
& \beta_{0}=1, \quad \beta_{1}=1, \quad \beta_{2}=1+\frac{1}{2} A, \quad \beta_{3}=\frac{1}{8} A^{2}+\frac{3}{4}(\alpha-R e)+1, \\
& \beta_{4}=\frac{1}{2} A^{2}+\frac{3}{8} A(\alpha-3 R e)+\frac{3}{2} \alpha-\frac{11}{4} R e+1, \quad \delta_{0}=0, \quad \delta_{1}=0 .
\end{aligned}
$$

As a result, the complete solution of (74) satisfying the boundary conditions in (14) can be obtained as follow:

$$
\begin{aligned}
f & =f^{(o)}+f^{(i)}-f^{(o) i} \\
& =1+\varepsilon^{\frac{1}{2}}\left\{-e^{-\xi}-\xi+1\right\}+\varepsilon\left\{e^{-\xi}\left(-\frac{1}{2} A \xi-\frac{1}{2} A-1\right)-\xi+\frac{1}{2} A+1\right\}+\varepsilon^{\frac{3}{2}}\left\{e ^ { - \xi } \left[\left(\frac{1}{4} \alpha+\frac{1}{4} R e\right.\right.\right. \\
& \left.\left.-\frac{1}{8} A^{2}\right) \xi^{2}+\left(-\frac{1}{4} \alpha+\frac{5}{4} R e-\frac{1}{8} A^{2}-\frac{1}{2} A\right) \xi+\left(-\frac{1}{4} \alpha+\frac{5}{4} R e-\frac{1}{8} A^{2}-A-1\right)\right]-\left(1+\frac{1}{2} A\right) \xi \\
& \left.+\left(\frac{1}{4} \alpha-\frac{5}{4} R e+\frac{1}{8} A^{2}+A+1\right)\right\}+\varepsilon^{2}\left\{e ^ { - \xi } \left[\left(-\frac{1}{4} A^{3}+\frac{1}{8} A^{2}\right) \xi^{3}+\left(-\frac{1}{8} A^{2}+\frac{5}{8} A R e-\frac{1}{8} A \alpha\right.\right.\right. \\
& \left.+\frac{1}{4} \alpha+\frac{1}{2} R e\right) \xi^{2}+\left(-\frac{3}{8} A^{2}-\frac{3}{8} A \alpha+\frac{9}{8} A R e-\frac{3}{4} \alpha+2 R e\right) \xi+\left(-\frac{1}{2} A^{2}-\frac{3}{8} A \alpha+\frac{9}{8} A R e-\frac{3}{2} \alpha\right. \\
& \left.\left.\left.+\frac{11}{4} R e-1\right)\right]-\left(\frac{1}{8} A^{2}+\frac{3}{4} \alpha-\frac{3}{4} R e+1\right) \xi+\frac{1}{2} A^{2}+\frac{3}{8} A(\alpha-3 R e)+\frac{3}{2} \alpha-\frac{11}{4} R e+1\right\} .
\end{aligned}
$$

As seen in Table 4, the asymptotic results agree well with the numerical ones no matter whether the parameters (i.e. $\alpha$ and $R e$ ) are positive or negative.

\subsection{Solution for large wall contraction ratios}

In this section, we take into account the leading influence of wall contraction ratio $\alpha$ on the flow, and treat $\varepsilon=-\frac{1}{\alpha}$ as the perturbation parameter. Then Eq.(13) becomes

$$
-\varepsilon f^{\prime \prime \prime}+\left(y f^{\prime \prime}+2 f^{\prime}\right)-\varepsilon R e\left(f f^{\prime \prime}-f^{\prime 2}\right)+\varepsilon M^{2} f^{\prime}=-\varepsilon \delta+2 \beta+\varepsilon R e \beta^{2}+\varepsilon M^{2} \beta,
$$

where $-\varepsilon \delta+2 \beta+\varepsilon R e \beta^{2}+\varepsilon M^{2} \beta=-\varepsilon k$. When $M=0$, Majdalani et al. [8] pointed out that the effect of large contraction ratio on flow behaviour was the same as that of large suction on flow behaviour. Thus, following the procedure outlined in Section 3.2, we introduce the stretching transformation of the form

$$
\xi=\frac{1-y}{\varepsilon}, \quad f(y)=1+\varepsilon g(\xi) .
$$


Then Eq.(97) becomes

$$
\varepsilon^{-1}(\dddot{g}+\ddot{g})-(\xi \ddot{g}+2 \dot{g}+\operatorname{Re} \ddot{g})-\varepsilon R e\left(g \ddot{g}-\dot{g}^{2}\right)-\varepsilon M^{2} \dot{g}=-\varepsilon \delta+2 \beta+\varepsilon R e \beta^{2}+\varepsilon M^{2} \beta,
$$

here denotes differentiation with respect to $\xi$. Substituting (79) into (99) and equating coefficients of $\varepsilon^{n}$ yields

$$
\begin{gathered}
\varepsilon^{-1}: \dddot{g}_{0}+\ddot{g}_{0}=0 \\
\varepsilon^{0}: \dddot{g}_{1}+\ddot{g}_{1}-(\xi+R e) \ddot{g}_{0}-2 \dot{g}_{0}-2 \beta_{0}=0 \\
\varepsilon: \ddot{g}_{2}+\ddot{g}_{2}-(\xi+R e) \ddot{g}_{1}-2 \dot{g}_{1}-\operatorname{Re}\left(g_{0} \ddot{g}_{0}-\dot{g}_{0}^{2}\right)-M^{2} \dot{g}_{0}=-\delta_{0}+2 \beta_{1}+\operatorname{Re} \beta_{0}^{2}+M^{2} \beta_{0}, \\
\varepsilon^{2}: \dddot{g}_{3}+\ddot{g}_{3}-(\xi+R e) \ddot{g_{2}}-2 \dot{g}_{2}-\operatorname{Re}\left(g_{0} \ddot{g}_{1}+g_{1} \ddot{g}_{0}-2 \dot{g}_{0} \dot{g}_{1}\right)-M^{2} \dot{g}_{1}=-\delta_{1}+2 \beta_{2}+2 \operatorname{Re} \beta_{0} \beta_{1}+M^{2} \beta_{1},
\end{gathered}
$$

The corresponding boundary conditions become

$$
g_{n}(0)=0, \quad \dot{g_{n}}(0)=0 ; \quad n=0,1,2, \cdots
$$

The outer solution $f^{(o)}=\beta y$ can be expressed in terms of the inner variable $\xi$ :

$$
f^{(o) i}=\left(\beta_{0}+\varepsilon \beta_{1}+\varepsilon^{2} \beta_{2}+\varepsilon^{3} \beta_{3}\right)-\varepsilon\left(\beta_{0}+\varepsilon \beta_{1}+\varepsilon^{2} \beta_{2}\right) \xi+\cdots \cdots
$$

By solving (100), one can obtain

$$
g_{0}=C_{1}\left(e^{-\xi}+\xi-1\right) .
$$

We can obtain $C_{1}=-1, \beta_{0}=1$, and $\beta_{1}=1$ using the matching process. Similarly, $g_{1}, g_{2}$ and $g_{3}$ can be derived as follows:

$$
\begin{gathered}
g_{1}=e^{-\xi}\left(-\frac{1}{2} \xi^{2}-R e \xi-C_{2}\right)+\left(R e-C_{2}\right) \xi+C_{2}, \\
g_{2}=e^{-\xi}\left[-\frac{1}{8} \xi^{4}-\frac{1}{2} R e \xi^{3}-\frac{1}{2}\left(R e^{2}+1\right) \xi^{2}+\left(-R e^{2}+2 R e+M^{2}\right) \xi-C_{3}\right]-\frac{1}{2} \delta_{0} \xi^{2}+\left(R e^{2}-2 R e\right. \\
\\
\left.\quad-M^{2}-C_{3}\right) \xi+C_{3}, \\
g_{3}=e^{-\xi}\left[-\frac{1}{48} \xi^{6}-\frac{1}{8} R e \xi^{5}+\left(-\frac{1}{4} R e^{2}+\frac{1}{8} R e-\frac{1}{8}\right) \xi^{4}+\left(-\frac{1}{6} R e^{3}+R e+\frac{1}{2} M^{2}\right) \xi^{3}+\left(-\frac{1}{2} R e^{3}+\frac{5}{2} R e^{2}\right.\right. \\
\left.\left.+\frac{7}{2} R e+R e M^{2}+M^{2}-\frac{1}{2}\right) \xi^{2}+\left(-R e^{3}+7 R e^{2}+9 R e+3 R e M^{2}+2 M^{2}\right) \xi+\frac{1}{4} R e-C_{4}\right] \\
-\frac{1}{4} e^{-2 \xi} R e-\frac{1}{2} \delta_{1} \xi^{2}+\left(R e^{3}-7 R e^{2}-\frac{37}{4} R e-3 M^{2} R e-2 M^{2}-C_{4}\right) \xi+C_{4},
\end{gathered}
$$

where $C_{2}=\beta_{2}=R e+1, C_{3}=\beta_{3}=R e^{2}-R e+1-M^{2}$ and $C_{4}=\beta_{4}=R e^{3}-7 R e^{2}-\frac{37}{4} R e-$ $3 M^{2} R e-2 M^{2}$. Finally, the complete solution of (97), subject to the boundary conditions in (14), 
can be expressed as

$$
\begin{aligned}
f & =f^{(o)}+f^{(i)}-f^{(o) i} \\
& =1+\varepsilon\left\{-e^{-\xi}-\xi+1\right\}+\varepsilon^{2}\left\{e^{-\xi}\left(-\frac{1}{2} \xi^{2}-R e \xi-R e-1\right)-\xi+R e+1\right\}+\varepsilon^{3}\left\{e ^ { - \xi } \left[-\frac{1}{8} \xi^{4}\right.\right. \\
& \left.-\frac{1}{2} R e \xi^{3}-\frac{1}{2}\left(R e^{2}+1\right) \xi^{2}+\left(-R e^{2}+2 R e+M^{2}\right) \xi-\left(R e^{2}-R e+1-M^{2}\right)\right]+(-R e-1) \xi \\
& \left.+\left(R e^{2}-R e+1-M^{2}\right)\right\}+\varepsilon^{4}\left\{-\frac{1}{4} e^{-2 \xi} R e+e^{-\xi}\left[-\frac{1}{48} \xi^{6}-\frac{1}{8} R e \xi^{5}+\left(-\frac{1}{4} R e^{2}+\frac{1}{8} R e-\frac{1}{8}\right) \xi^{4}\right.\right. \\
& +\left(-\frac{1}{6} R e^{3}+R e+\frac{1}{2} M^{2}\right) \xi^{3}+\left(-\frac{1}{2} R e^{3}+\frac{5}{2} R e^{2}+\frac{7}{2} R e+R e M^{2}+M^{2}-\frac{1}{2}\right) \xi^{2}+\left(-R e^{3}\right. \\
& \left.\left.+7 R e^{2}+9 R e+3 R e M^{2}+2 M^{2}\right) \xi+-R e^{3}+6 R e^{2}+\frac{21}{2} R e+3 M^{2}(R e+1)-1\right]+\left(-R e^{2}\right. \\
& \left.\left.+R e+M^{2}-1\right) \xi+\left(R e^{3}-6 R e^{2}-\frac{41}{4} R e-3 M^{2} R e-3 M^{2}+1\right)\right\} .
\end{aligned}
$$

A comparison of $-f^{\prime \prime}(1)$ between the asymptotic and numerical solutions is shown in Table 5. When $\alpha$ is large, the asymptotic results have a good match with the numerical results.

\section{Comparison of the numerical and asymptotic solutions and discussion}

The main aim of this section is to present a comparison of the numerical and asymptotic solutions and some discussion. For the former, the BVP (i.e. (13) and (14)) has to be solved numerically. Here we use bvp4c of Matlab to solve it [45]. In addition, unless stated otherwise, for all our computations with bvp4c, we use the default relative error tolerance $10^{-3}$ and the default absolute error tolerance $10^{-6}$. On the other hand, we will compare asymptotic results with numerical results to verify the accuracy of asymptotic solutions constructed in Section 3. A number of possible ways on the comparison are available, e.g. by comparing $f(y), f^{\prime}(y)$ proportional to the flow velocity, $f^{\prime \prime}(1)$, related to the skin-friction at the walls, etc. In [46] Terrill pointed out that the comparison of $f^{\prime \prime}(1)$ was found to be the most effective way. Here we will follow his line, and the corresponding values of $-f^{\prime \prime}(1)$ with the different ranges of the control parameters are presented in Tables 1-5.

To develop a better understanding of the flow character, in the following section we not only graphically show the axial velocity profiles $f^{\prime}(y)$ over different ranges of the control parameters $\alpha, R e$ and $M$, but also further give some discussion on the effects of these parameters on flow behavior.

When $R e=600$, Fig.2 illustrates the behaviour of the self-similar axial velocity $f^{\prime}(y)$ for expansion ratio $\alpha=-2$ and 2 , respectively, over a range of dimensionless Hartmann number $M$. In Fig.2a, an initial glance indicates that the effect of varying $M$ on the axial velocity $f^{\prime}(y)$ is

not obvious. That is because for this case, the incompressible fluid injecting from the walls of the channel is mainly to determine the flow behaviour in comparison with the magnetic field and the wall deformation. When the Reynolds number is increased, the axial velocity distribution approaches a cosine profile (see (41) in Section 3), specifically

$$
f^{\prime}(y)=\cos \left(\frac{\pi}{2} y\right) ; \quad R e \rightarrow+\infty
$$

(111) has often been called "Taylor's profile" due to its relevance to several applications including 
paper manufacture and gas separation. With the comparison of asymptotic and numerical solutions, an error appears with Hartmann number $M$. Also the error increases as $M$ increases. That is because the asymptotic solution constructed in Section 3.1 is only suitable for small Hartmann number (i.e. $M^{2}=O(1)$ ), which can be verified by the case $M=0$. Meanwhile, as seen in Fig.2a, the largest error seems to occur near the center of the channel for a fixed $M$. When $\alpha=2$, similar conclusions can be drawn (see Fig.2b).

Fig.3 presents the self-similar axial velocity $f^{\prime}(y)$ for large suction. In this case, since sufficiently large suction can dominate over wall expansion and magnetic filed, the suction region formed near the closed head-end causes the incompressible fluid entering from the right side of the channel to move farther upstream to the walls along the wall-normal direction. Here the non-slip condition (i.e. $f^{\prime}(1)=0$ in $(14)$ ) is also considered by us. As a result, the axial velocity profile becomes a spatially uniform value 1 near the center of the channel while appearing a thinning boundary layer near the walls of the channel. Moreover, from (43) in Section 3.2, we can obtain that the boundary layer has a thickness of $O(\varepsilon)$. In addition, in Fig.3, we also see the comparison between numerical and asymptotic solutions for $f^{\prime}(y)$. The magnified graph indicates that a better agreement can be realized as $M \rightarrow 0$, and the error increases as $M$ increases. However, on the whole, the accuracy of the asymptotic solution (i.e. (64) in Section 3.2) is reliable. by recalling (64) and (73), the asymptotic solution ultimately collapses into the essentially irrotational form, namely,

$$
f(y)=y+O\left(R e^{-1}\right) ; \quad R e \rightarrow-\infty .
$$

Such behaviour is also consistent with Majdalani et al. [9] and Hang Xu et al. [11] in the absence of magnetic field.

To study the self-similar axial velocity sensitivity to $M$, the expansion ratio is held constant at $\alpha=-2$ and $\alpha=2$ for a fixed $R e=1$ (see Fig.4). When the walls of the channel were motionless, Terrill and Shrestha [22] pointed out that a magnetic boundary layer existed near the walls if $R e$ was small and $M$ was large. Further if $R e(<0)$ and $M$ were both large, then the flow near the walls would consist of a combined magnetic and suction boundary layer. As predicted by them, here we do find the existence of a magnetic boundary layer (see $M=200$ ). Moreover, considering the balance between viscous and magnetic terms, we also obtain that the thickness of the magnetic boundary layer is $O\left(\varepsilon^{\frac{1}{2}}\right)$, which is different from the result included in Section 3.2. In addition, for $M=200$, when $R e$ is varied from 1 to 20, both numerical ( -$)$ and asymptotic $(\cdots)$ solutions are compared. The results are found to be in very well agreement, which indicates that the accuracy of the asymptotic solution (96) is reliable.

When $R e=1,15$ and $M=0$, for varying $\alpha$, the corresponding self-similar axial velocity profiles are plotted in Fig.5. It may be interesting to note that the special case $M=0$ and $\alpha=0$, where the value of $f^{\prime}(0)$ is near 1.5 and the self-similar axial velocity profile is parabolic. Such behaviour is consistent with one in the fully developed Hagen-Poiseuille flow. As $\alpha$ is varied from 0 to -200 , a boundary layer is gradually formed. That is because the rapid volumetric contraction of the walls causes the incompressible fluid near the closed head-end to rapidly move towards the right side of the channel. When comparing Fig.3 with Fig.5, we can conclude that they have some similar phenomena, e.g. from (98), the thickness of the boundary layer is $O(\varepsilon)$, and is equal to one for large suction. when $M=0$, to describe the similar effects of large suction and large contraction on flow behaviour, Majdalani et al. [8] have even defined an effective suction Reynolds number, that is,

$$
R_{s}=-(R e+\alpha)
$$


which combined the effect of the suction with the one of the wall contraction. On the other hand, for $\alpha=-200$, as $M$ is varied from 0 to 20, both numerical ( -$)$ and asymptotic $(\cdots)$ solutions are compared. Obviously, the accuracy of the asymptotic solution (110) is reliable, and the precision extends to 3 significant figures.

\section{Conclusions}

In this paper, for the BVP (13) and (14), we have constructed asymptotic solutions for different ranges of the control parameters. Based on numerical solutions with bvp4c, the accuracy of these asymptotic solutions is also easily verified. On the other hand, by discussing the effects of the control parameters on the flow behaviour, some conclusions may be summarized as follows:

i) For large injection, the axial velocity profile is still parabolic when $M^{2}=O(1)$.

ii) The Lighthill method has been successfully used to eliminate the singularity of the higher order derivatives.

iii) Whether the situation is large suction, large Hartmann number or large contraction ratio, the boundary layer can always be found, but the corresponding thicknesses are different. 


\section{Acknowledgments}

This work is partially supported by the National Natural Science Foundations of China (Nos. 91430106, 11302024), Fundamental Research Funds for the Central Universities (Nos: 06108038, FRF-BR-13-023, FRF-TP-15-036A3), and the foundation of the China Scholarship Council in 2014(File No.154201406465041).

\section{References}

[1] N. Srivastava, Analysis of flow characteristics of the blood flowing through an inclined tapered porous artery with mild stenosis under the influence of an inclined magnetic field, J. Biophys. 24 (2014) $1-9$.

[2] S.L. Wilens, The experimental production of lipid deposition in excised arteries, Science. 114 (1951) 389-393.

[3] S.M. Evans, H.K. Thrig, J.A. Means, W. Zeit, E.R. Haushalter, Atherosclerosis: an in vitro study of the pathogenesis of atherosclerosis, Am. J. Clin. Pathol. 22 (1952) 354-363.

[4] C.C. Michel, Direct observations of sites of permeability for ions and small molecules in mesothelium and endothelium, In Capillary Permeability. (1953) 628-642.

[5] J.C. Misra, S.K. Ghosh, A mathematical model for the study of blood flow through a channel with permeable walls, Acta. Mech. 122 (1997) 137-153.

[6] J.C. Misra, A. Sinha, G.C. Shit, A numerical model for the magnetohydrodynamic flow of blood in a porous channel, J. Mech. Med. Biol. 11 (2011) 547-558.

[7] M. Goto, S. Uchida, Unsteady flows in a semi-infinite contracting or expanding pipe with a porous pipe, In: Proceedings of the 40th Japan National Congress Applied Mechanics, Tokyo, Japan. (1990) 163-172.

[8] E.C. Dauenhauer, J. Majdalani, Exact self-similarity solution of the Navier-Stokes equations for a porous channel with orthogonally moving walls, Phys. Fluids. 15 (2003) 1485-1495.

[9] J. Majdalani, C. Zhou, Moderate-to-large injection and suction driven channel flows with expanding or contracting walls, Z. Angew. Math. Mech. 83 (2003) 181-196.

[10] S. Asghar, M. Mushtaq, T. Hayat, flow in a slowly deforming channel with weak permeability: An analytical approach, NONLINEAR Anal-Real, 11 (2010) 555-561.

[11] H. Xu, Z.L. Lin, S.J. Liao, J.Z. Wu, J. Majdalani, Homotopy-based solutions of the Navier-Stokes equations for a porous channel with orthogonally moving walls, Phys. Fluids. 22 (2010) 053601-053618.

[12] V.I. Karmilov, Biological and therapeutic action of a magnetic field and strictly periodic vibration, Exp. Clin. Material. Russian.

[13] R. Sambasiva, Numerical solution of non-steady magnetohydrodynamic flow of blood through a porous channel, J. Biomed. Eng. 10 (1988) 293-295. 
[14] T. Higashi, A. takeuchi, N. Kawaguchi, S. Sagawa, S. Onishi, M. Date, Orientation of erythrocytes in a strong static magnetic field, J. Blood, 82 (1993) 1328-1334.

[15] A. Kolin, Electromagnetic flow meter: principle of method and its applications to blood flow measurement, in proceedings of the society for experimental biology and medicine, 35 (1936) 53-56.

[16] E.M. korchevskii, L.S. Marochnik, Magnetohydrodynamic version of movement of blood, Biophysics, 10 (1965) 411-414.

[17] V.A. Vardanyan, Effect of a magnetic field on blood flow, Biophysics, 18 (1973) 515-521.

[18] V.K. Sud, P.K. Suri, R.K. Mishra, Effect of magnetic field on oscillating blood flow in arteries, Studia Biophysica. 46 (1974) 163-171.

[19] S. Chandrasekhar, Hydrodynamic and hydromagnetic stability, Oxford University Press London.

[20] N. Rudraiah, Magnetohydrodynamic stability of heterogeneous incompressible non-dissipative conducting liquids, J. Appl. Sci. Res. 11 (1962) 105-117.

[21] U. Suryaprakasarao, Laminar flow in channels with porous walls in the presence of a transverse magnetic field, J. Appl. Sci. Res. 9 (1961) 374-382.

[22] R.M. Terrill, G.M. Shrestha, Laminar flow through channels with porous walls and with applied transverse magnetic field, J. Appl. Sci. Res. 11 (1963) 134-144.

[23] R.M. Terrill, G.M. Shrestha, Laminar flow in a uniformly porous channel with an applied transverse magnetic field, J. Appl. Sci. Res. 12 (1964) 203-211.

[24] A.S. Berman, Laminar flow in channels with porous walls, J. Appl. Phys. 24 (1953) 1232-1235.

[25] W.A. Robinson, The existence of multiple solutions for the laminar flow in a uniformly porous channel with suction at both walls, J. Eng. Math. 10 (1976) 23-40.

[26] F.M. Skalak, C.Y. Wang, On the nonunique solutions of laminar flow through a porous tube or channel, SIAM J. Appl. Math. 34 (1978) 535-544.

[27] K.G. Shih, On the existence of solutions of an equation arising in the theory of laminar flow in a uniformly porous channel with injection, SIAM J. Appl. Math. 47 (1987) 526-533.

[28] M.C. Stephen, Analysis of steady flow in a channel with one porous wall, or with accelerating walls, SIAM J. Appl. Math. 15 (1991) 429-438.

[29] C.Q. Lu, A.D. MacGillivray, S.P. Hastings, Asymptotic behavior of solutions of a similarity equation for laminar flows in channels with porous walls, IMA J. Appl. Math. 49 (1992) 139-162.

[30] A.D. MacGillivray, C.Q. Lu, Asymptotic solution of a laminar flow in a porous channel with large suction: a nonlinear turning point problem, J. MAA. 1 (1994) 229-248. 
[31] C.Q. Lu, On the asymptotic solution of laminar channel flow with large suction, SIAM J. Math. Anal. 28 (1997) 1113-1134.

[32] C.Q. Lu, On the uniqueness of laminar channel flow with injection, J. Appl. Anal. 73 (1999) 497-505.

[33] C.Q. Lu, On matched asymptotic analysis for laminar channel flow with a turning point. Conference on differential equations and computational simulations. 3 (1999) 109-118.

[34] J.F. Brady, Flow development in a porous channel or tube, Phys. Fluids. 27 (1984) 1061-1067.

[35] L. Durlofsky, J.F. Brady, The spatial stability of a class of similarity solutions, Phys. Fluids. 27 (1984) 1068-1076.

[36] J. Sobey, G. Drazin, Bifurcations of two-dimensional channel flows, J. Fluid Mech. 171 (1986) 263-287.

[37] M.B. Zaturska, P.G. Drazin, W.H.H. Banks, On the flow of a viscous fluid driven along a channel by suction at porous walls, Fluid Dyn. 4 (1988) 151-178.

[38] C. Zhou, J. Majdalani, Inner and outer solutions for the injection driven channel flow with retractable walls, 33rd AIAA Fluid Dynamics Conference, (2003).

[39] S.W. Yuan, Further investigation of laminar flow in channels with porous walls, J. Appl. Phys. 3 (1956) 267-369.

[40] R.M. Terrill, Laminar flow in a uniformly porous channel with large injection, The Aeronautical Quarterly, 16 (1965) 323-332.

[41] M.V. Dyke, Perturbation methods in fluid mechanics, Annotated Edition, Stanford: Parabolic Press (1975).

[42] Y.C. Su, Boundary layer correction method for singular perturbation problems, Shanghai Science and Technology Press, 1983.

[43] M.I. Vishik, L.A. Lyusternik, Regular degeneration and boundary layer for linear differential equations with small parameter, Uspekhi Matematicheskikh Nauk. 3 (1957).

[44] M.I. Vishik, L.A. Lyusternik, The solution of some perturbation problems for matrices and selfadjoint or non-selfadjoint differential equations, Russ. Math. Surv. 3 (1960).

[45] L.F. Shampine, J. Kierzenka, M.W. Reichelt, Solving boundary value problems for ordinary differential equations in MATLAB with bvp4c, (2000).

[46] R.M. Terrill, G.M. Shrestha, Laminar flow through parallel and uniformly porous walls of different permeability, Z. Angew. Math. Phys. 16 (1965) 470-482. 
List of Figures

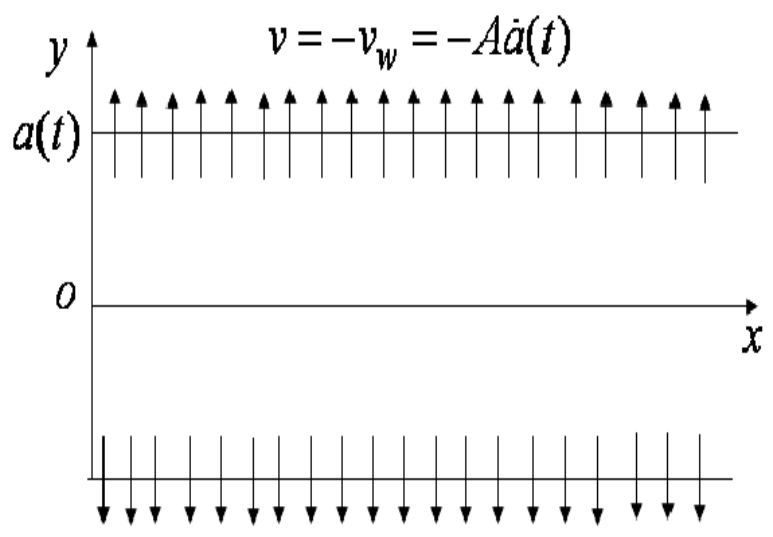

Figure 1: Physical Configuration
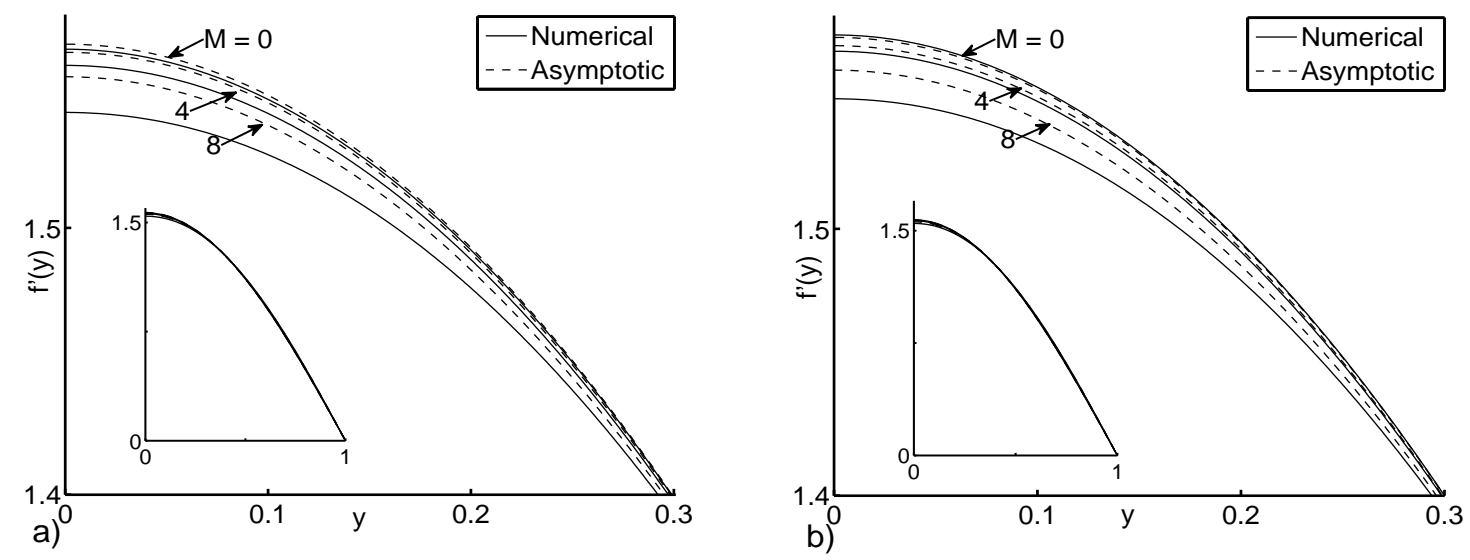

Figure 2: Comparison between numerical and asymptotic solutions for $f^{\prime}(y)$ at a) $\alpha=-2$, and b) $\alpha=2 ; R e=600$. 

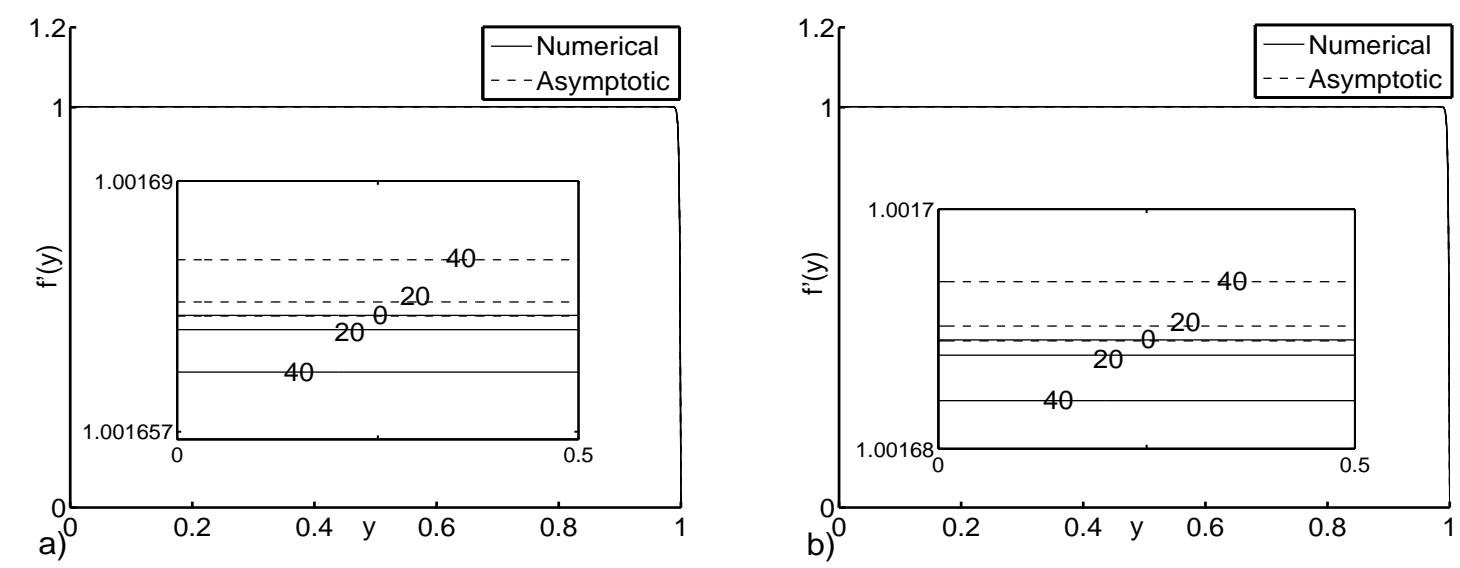

Figure 3: Comparison between numerical and asymptotic solutions for $f^{\prime}(y)$ at a) $\alpha=-2$, and b) $\alpha=2$; $R e=-600$.
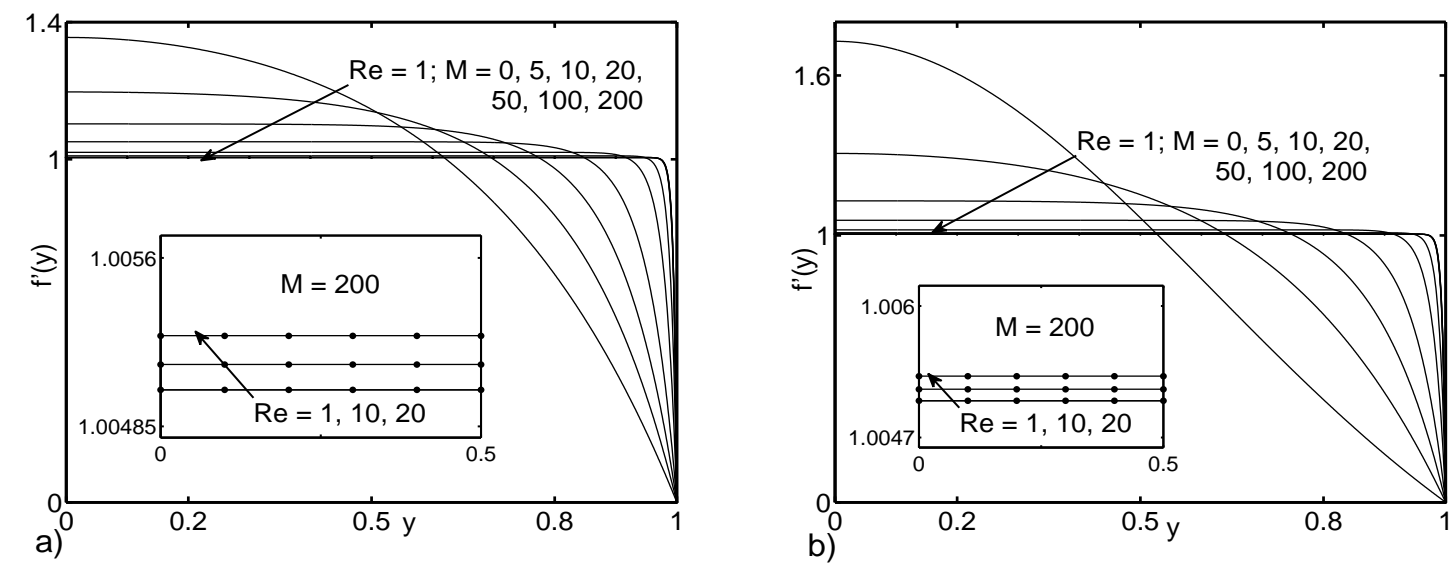

Figure 4: Comparison between numerical and asymptotic solutions for $f^{\prime}(y)$ at a) $\alpha=-2$, and b) $\alpha=2$. 

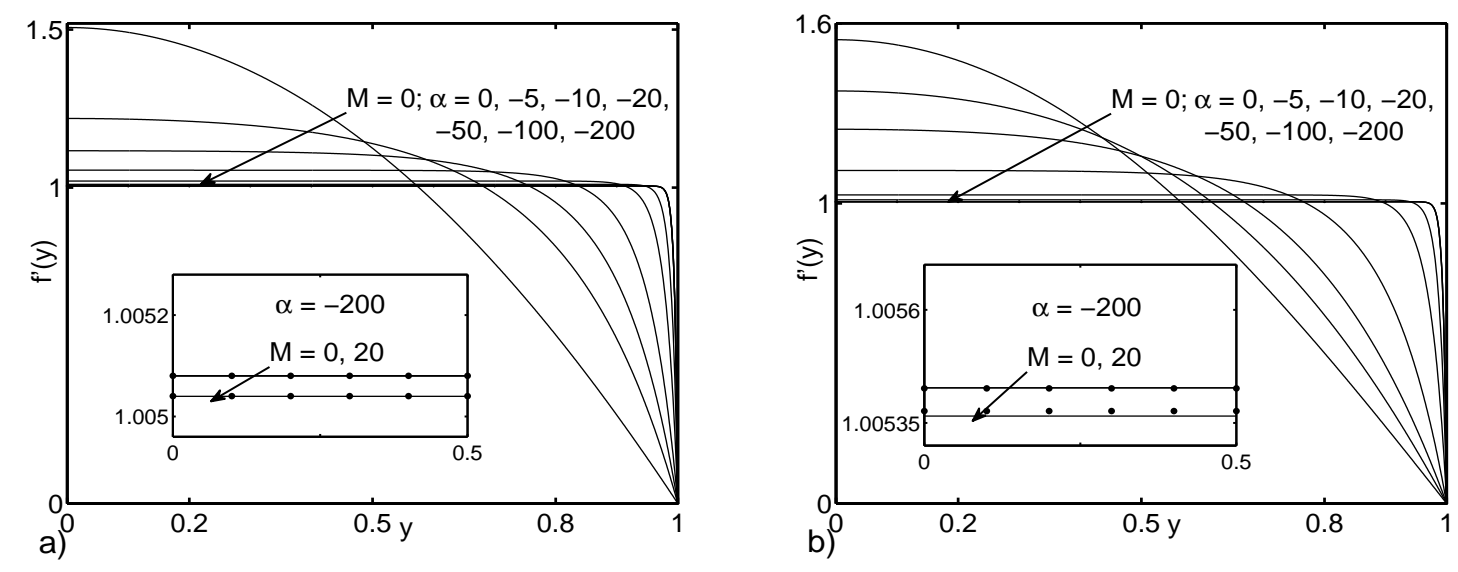

Figure 5: Comparison between numerical and asymptotic solutions for $f^{\prime}(y)$ at a) $R e=1$, and b) $R e=15$. 